\title{
WILEY-VCH
}

DOI: 10.1002/ ((please add manuscript number))

Article type: (Full Paper)

\section{Influence of the nature of A cation on dynamics of charge transfer processes in perovskite solar cells}

Pankaj Yadav, ${ }^{*}$ Mohammad Alotaibi, ${ }^{*}$ Neha Arora, M. Ibrahim Dar, ${ }^{*}$ Shaik Mohammed Zakeeruddin, and Michael Grätzel

Dr. P. Yadav, Dr. N. Arora, Dr. M. I. Dar, Dr. S. M. Zakeeruddin, Prof. M. Grätzel Laboratory of Photonics and Interfaces, Institute of Chemical Sciences and Engineering, École Polytechnique Fédérale de Lausanne, CH-1015-Lausanne, Switzerland.

Dr. M. Alotaibi

National Nanotechnology center, King Abdulaziz city for science and technology, Riyadh, Saudi Arabia.

E-mail: ibrahim.dar@epfl.ch

†P.Y. and M.A. contributed equally to this work.

Keywords: (perovskite solar cell, organic cation, interfacial dynamics, electrochemical impedance spectroscopy, current voltage characteristics)

The electronic processes occurring within the perovskite solar cells (PSCs) are strongly influenced by the nature of the organic A-cations present within the inorganic framework. In the present study, we investigated the impact of FA $\left(\mathrm{CH}\left(\mathrm{NH}_{2}\right)_{2}{ }^{+}\right)$and $\mathrm{Cs}^{+}$cations on the intrinsic and interfacial properties in the $\mathrm{FAPbBr}_{3}$ and $\mathrm{CsPbBr} 3$ PSCs. The analysis of current density ( $\left.\mathrm{J}_{\mathrm{SC}}\right)$ and photovoltage ( $\mathrm{V}_{\mathrm{OC}}$ ) as a function of illumination intensity establishes that the interfacial charge transport is more rapid in $\mathrm{FAPbBr}_{3}$ devices. Small perturbation measurements, including intensity modulated photocurrent and photovoltage spectroscopy were applied to explore the resistive and capacitive elements. Furthermore, electrochemical impedance spectroscopy measurements were found to correlate well with the photovoltaic characteristics of $\mathrm{FAPbBr}_{3}$ and $\mathrm{CsPbBr}_{3}$ PSCs. Overall, the in-depth analysis of various phenomena occurring within the bromide PSCs allowed us to underline the working principle 


\section{WILEY-VCH}

which provides a key to optimize the device performance. The present protocol is not only valid for PSCs but can also be extended to devices based on alternative light harvesters.

\section{Introduction}

Among next generation photovoltaic (PV) technologies, perovskite solar cells (PSCs) have become a center of interest due to the low-cost solution processability. ${ }^{[1]}$ A typical perovskite PV light harvester, achieving high power conversion efficiency (PCE), contains organic cations, like methylamonium $\left(\mathrm{MA}=\mathrm{CH}_{3} \mathrm{NH}_{3}{ }^{+}\right)$and formamidinium $\left(\mathrm{FA}=\mathrm{CH}\left(\mathrm{NH}_{2}\right)_{2}{ }^{+}\right)$within the lead halide inorganic framework. ${ }^{[2]}$ Despite the remarkable advancement, combatting stability issues particularly in humid and hot conditions has remained a challenge. ${ }^{[3]}$ In addition to organic part, above mentioned stability issues are also associated with the inorganic framework.

Both inherent issues could be addressed by using a combination of lead bromide framework and less polar or spherical cations, such as $\mathrm{CH}\left(\mathrm{NH}_{2}\right)_{2}{ }^{+}(\mathrm{FA})$ or cesium $\left(\mathrm{Cs}^{+}\right)$. $\mathrm{FAPbBr}_{3}$ and $\mathrm{CsPbBr}_{3}$ exhibit, respectively, band gap of 2.27 and $2.35 \mathrm{eV}$, which are desired to obtain high $V_{O C .}{ }^{[4,5]}$ Along with higher stability, bromide based PSCs also possess a high open circuit voltage $\left(V_{O C}\right)$ of $>1.3 \mathrm{~V}$, enabling them to be directly applicable for photocatalytic water splitting and to harvest high energy of the solar spectrum in a tandem configuration with the matured PV technologies. ${ }^{[4,5]}$ From these reports, the difference in the photovoltaic characteristics seems to be associated with the nature of cations. However, there is a scarcity of reports in the literature discussing the role of organic cations on the intrinsic and interfacial dynamics of PSC. In this work, we analyzed the PSCs based on $\mathrm{FAPbBr}_{3}$ and $\mathrm{CsPbBr}_{3}$ to understand the recombination losses, capacitive characteristics, and charge transport and extraction within the absorber layer and across various interfaces. We studied current density $\left(J_{S C}\right)$, and open-circuit voltage $\left(V_{O C}\right)$ as a function of illumination to explore the charge transport and dominant recombination mechanism within the devices. The intensity 


\section{WILEY-VCH}

modulated photocurrent and photovoltage spectroscopy (IMPS/IMVS) and electrochemical impedance spectroscopy (EIS) were further exploited to identify the dominant recombination process and transport limiting factors. Detailed analysis of the physical phenomena occurring in the $\mathrm{FAPbBr}_{3}$ and $\mathrm{CsPbBr}_{3}$ PSCs allowed us to underline the working principle.

\section{Results and Discussions}

The current density versus voltage (J-V) characteristic measured under AM1.5G (light intensity of $100 \mathrm{~mW} / \mathrm{cm}^{2}$ ) at $25^{\circ} \mathrm{C}$ for $\mathrm{CsPbBr}_{3}$ (device A) is shown in Figure 1a. Power conversion efficiency $(P C E)$ of $2.0 \%$ with an open circuit voltage $\left(V_{O C}\right)$ of $1.22 \mathrm{~V}$, fill factor $(F F)$ of $65 \%$ and short circuit current density $\left(J_{S C}\right)$ of $2.42 \mathrm{~mA} / \mathrm{cm}^{2}$ is recorded for the $\mathrm{CsPbBr} 3$ device. The replacement of $\mathrm{Cs}$ with FA in the lattice (device $\mathrm{B}$ ) resulted in a notable difference in the values of $P C E(6.19 \%), V_{O C}(1.45 \mathrm{~V}), J_{S C}\left(5.97 \mathrm{~mA} / \mathrm{cm}^{2}\right)$ and $F F(70 \%)$. The increase in PCE is mainly due to higher $F F$ and $V_{O C}$, which could arise from the reduction of electron and hole transport resistances, higher shunt resistance and less non-radiative recombination. However, in the present case, the electron and hole transport layer i.e. $\mathrm{TiO}_{2}$ and spiro-OMeTAD are deposited under similar experimental conditions. Thus, the improvement in the charge transport and decrease in the trap-assisted recombination could be associated with the absorber material. The semi-logarithmic plots of dark J-V characteristics shown in Figure $1 \mathrm{~b}$ for both devices exhibit two distinguishable bias dependent regions. In case of device $\mathrm{B}$, the low forward bias region is dominated by higher shunt resistance which reflects the formation of a better rectification junction with a low leakage current between the selective contacts and absorber layer. ${ }^{[6]}$ In the high forward bias region at $V>0.8 \mathrm{~V}$, for both devices, the net current plots are almost parallel and follow an exponential behavior due to the diode and recombination characteristics. The main difference lies in the lower order magnitude of net current in device B arising from the better interfacial junction and absorber 


\section{WILEY-VCH}

quality. Figure 2a shows the linear dependence of $J_{S C}$ on the incident light intensity, with a fit to a power law $\left(I \propto J_{S C}{ }^{\alpha}\right)$, resulting in the value of $\alpha \approx 1$ for both devices. ${ }^{[7]}$ To draw the conclusion about a dominant recombination mechanism, knowledge about the upper limit of Fermi level splitting is required as this will allow us to distinguish between charge separation and charge storage dynamics. An independent illustration of dominant recombination can be drawn from Voc vs illumination intensity plot; an alternative way to obtain Fermi level splitting.
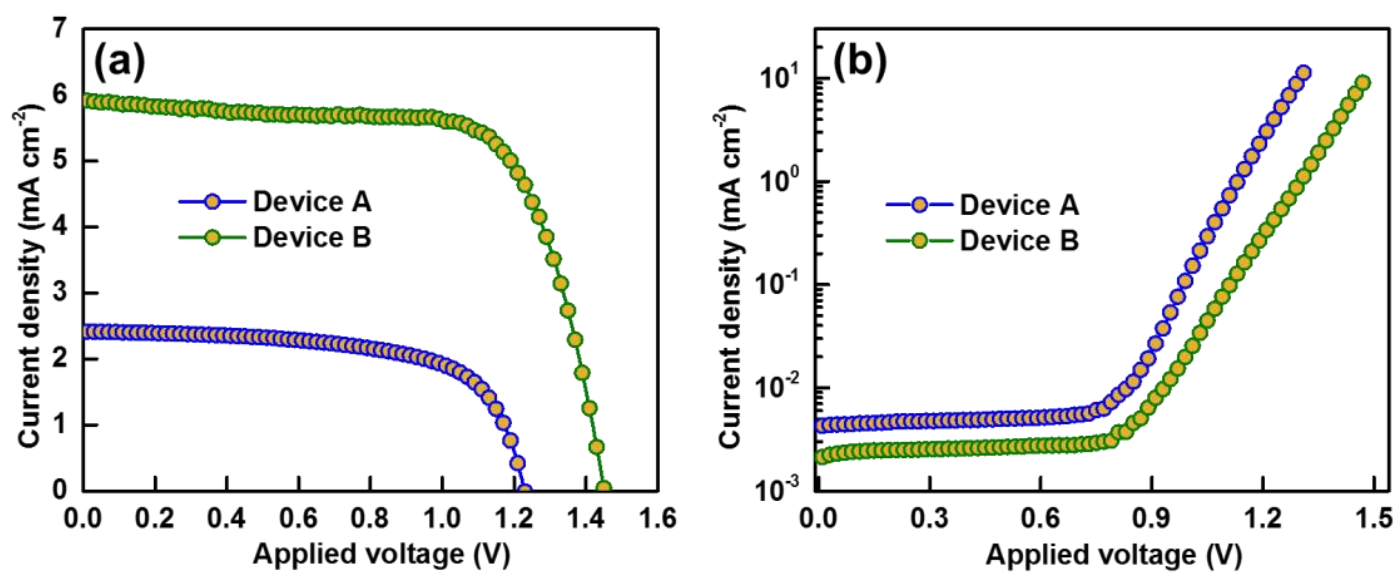

Figure 1. Photovoltaic characteristics of $\mathrm{CsPbBr}_{3}$ (device A) and $\mathrm{FAPbBr}_{3}$ (device $\mathrm{B}$ ) perovskite solar cells. (a) Current density versus voltage characteristics of $\mathrm{CsPbBr}_{3}$ (device $\mathrm{A}$ ) and $\mathrm{FAPbBr}_{3}$ (device $\mathrm{B}$ ) devices recorded under full sun illumination at a scan speed of 0.01 $\mathrm{Vs}^{-1}$ in the backward direction, (b) Current density versus voltage characteristics of $\mathrm{CsPbBr} 3$ (device $\mathrm{A}$ ) and $\mathrm{FAPbBr}_{3}$ (device $\mathrm{B}$ ) devices recorded in the dark.

Figure $2 \mathrm{~b}$ shows the semi-logarithmic plot of $V_{O C}$ vs illumination intensity for both devices and a straight line fit using the expression $V_{O C} \propto \frac{n k T}{q} \log _{10} I$, where $q$ is the electric charge, $n$ is the ideality factor, $k$ is the Boltzmann's constant and $T$ is the temperature. The deviation of the slopes from $2 k_{B} T / q$ illustrates the dominance of monomolecular recombination close to the $V_{O C}$ of devices. Relatively lower ideality factor of 1.7 in the case of device B (1.9 in the case of device A) indicates a reduced trap-assisted recombination 


\section{WILEY-VCH}

which could be related to quality of the absorber layer and interfaces of the device. ${ }^{[7-8]}$ Hence, the findings of $J_{S C}$ and $V O C$ vs intensity suggest that the performance of device A is limited by the recombination mechanism. In addition to the recombination process, it is important to evaluate the transport characteristics under operating conditions.
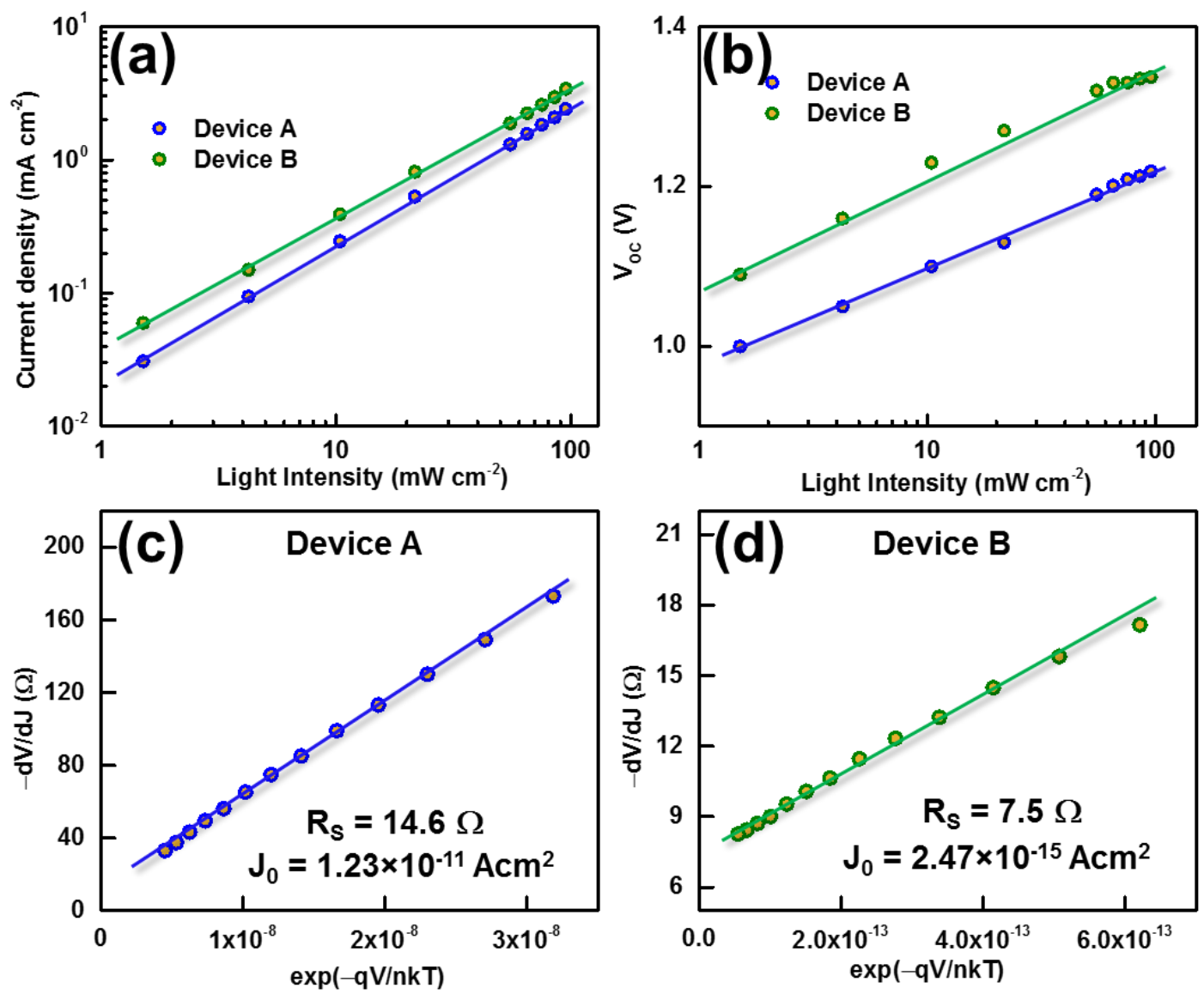

Figure 2. The dependence of (a) $J_{S C}$ and (b) $V_{O C}$ on the incident light intensity in case of $\mathrm{CsPbBr} 3$ (device A) and $\mathrm{FAPbBr}_{3}$ (device B) solar cells. The plot of $-d V / d J$ vs $\exp (-q V / n k T)$ was used to extract the values of $R_{S}$ and $J_{0}$, here $\mathrm{V}$ is applied voltage corrected for IRs losses for (c) $\mathrm{CsPBr}_{3}$ (device A), and (d) $\mathrm{FAPbBr}_{3}$ (device B) solar cells under illumination.

Figure $2 \mathrm{c}$ and $\mathrm{d}$ illustrate the plot of the measured $-d V / d J$ close to the open circuit voltage $\left(V_{O C}\right)$ under full-sun illumination for the device A and B, respectively. The obtained plot is fitted using the expression $d V / d J=R_{S}+\frac{n k T}{q J_{0}} e^{-(q V / n k T)}$ where, $J_{0}$ represents the reverse saturation current. The intercept of straight line and the slope, yield $R_{S}$ and $J_{0}$, 


\section{WILEY-VCH}

respectively. The value of $n$ obtained from the $V_{O C}$ vs illumination intensity plot (Figure 2b) is used as an input parameter in the above expression. $R_{S}$ of 14.5 and $7.5 \Omega$ were obtained under illumination, respectively, for the device A and B. Relatively lower $R S$ obtained in case of device $\mathrm{B}$ relates to the specific conductivity of $\mathrm{FAPbBr} 3$ semiconductor or more favorable band alignment of the absorber with the electron and hole transport layers. ${ }^{[9]}$ Furthermore, we studied the charge transport and recombination time for both devices using small perturbation techniques, i.e., IMPS, IMVS and EIS, which have been widely used for characterizing dyesensitized and silicon solar cells, and recently for PSCs. Nevertheless, there is still dearth of reports demonstrating their application in the bromide PSCs. As bromide based PSCs possess different electrical and optical characteristics than the conventional iodide based PSCs, it would be interesting to study their transport and recombination characteristics using such techniques.

Figure 3 shows the IMPS and IMVS response of both devices in the frequency range of $100 \mathrm{kHz}$ to $1 \mathrm{~Hz}$ at a perturbation current of $15 \mathrm{~mA}$. At a fixed illumination, the IMPS response of the devices features a Warburg element in the low and mid frequency region followed by a straight line in the high frequency region. A distinct feature in the low and mid frequency is ascribed to two different transport processes running in parallel within the device. Giordano et al. and Guillen et al. observed a similar IMPS features for lithium treated and untreated $\mathrm{TiO}_{2}$ photoanodes and concluded that the low and mid frequency features are associated with the charge transport processes in the $\mathrm{TiO}_{2}$ and perovskite layer, respectively. ${ }^{[10]}$ The transport time can be obtained directly from the minimum frequency $\left(f_{\min }\right)$ point of the complex Nyquist spectra by using the expression $\tau_{n}=1 / 2 \pi f_{\min } \cdot \tau_{n}$ values of 5 and 0.8 ms were obtained, respectively, for the device A and B. A faster time response in device $\mathrm{B}$ than device $\mathrm{A}$ signifies better charge transport kinetics at the $\mathrm{TiO}_{2} /$ perovskite interface. The Nyquist IMPS spectra recorded under different illuminations for both devices 


\section{WILEY-VCH}

are shown in Figure S1. With an increase in the illumination, the mid and low frequency spectra merge, and a complex spectrum at the low frequency followed by the Warburg element and a high frequency line appeared. The appearance of the Warburg element in the bromide based PSC signifies the combined influence of the electron transport in $\mathrm{TiO}_{2}$ and the recombination within the active layer.
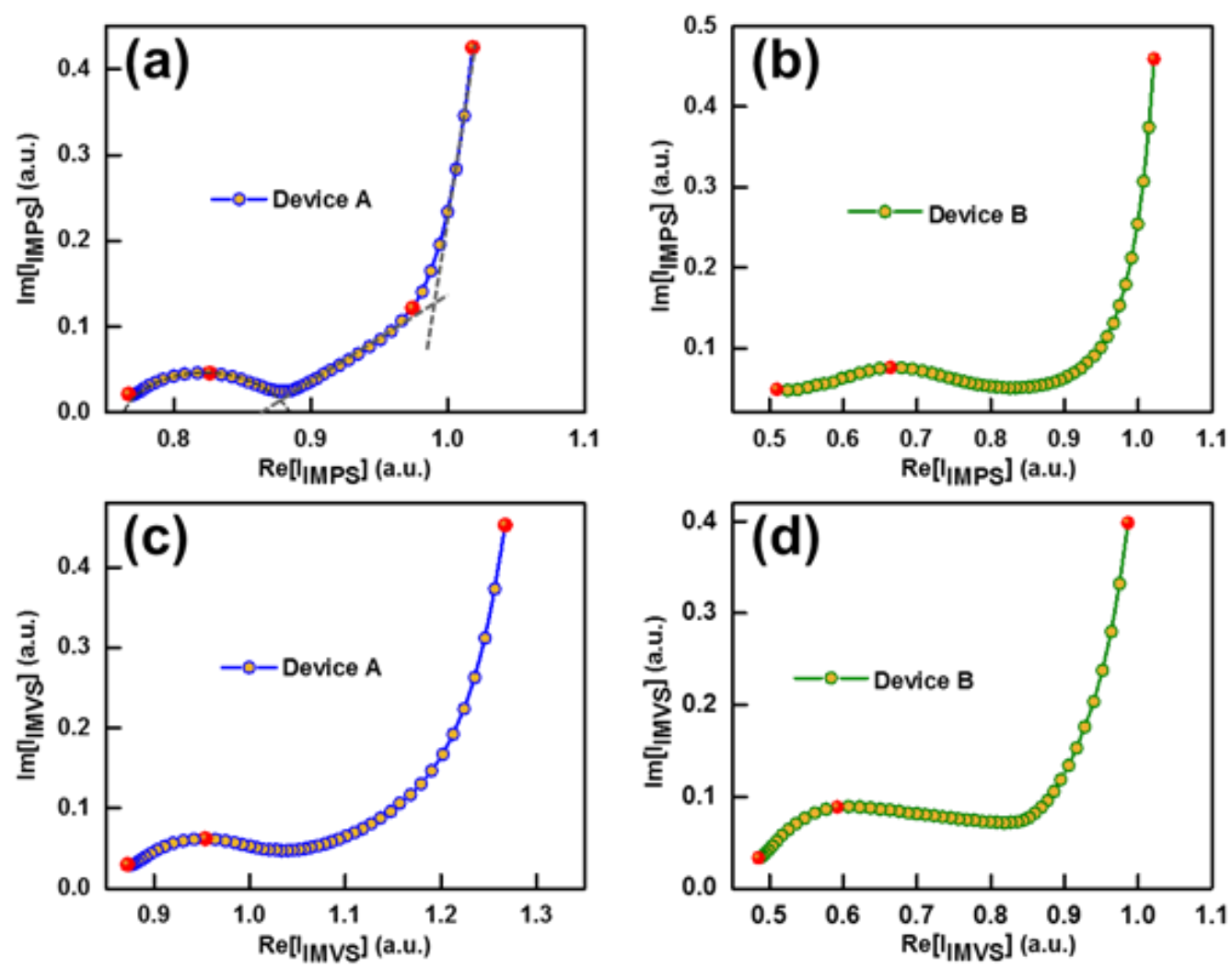

Figure 3. IMPS response of (a) $\mathrm{CsPbBr}_{3}$ (device $\mathrm{A}$ ) and (b) $\mathrm{FAPbBr}_{3}$ (device $\mathrm{B}$ ) solar cells, and IMVS response of (c) $\mathrm{CsPbBr}_{3}$ (device A) and (d) $\mathrm{FAPbBr}_{3}$ (device B) solar cells recorded in the frequency range of $100 \mathrm{kHz}$ to $1 \mathrm{~Hz}$.

To get further insight into the effective charge transport in the devices, current-time $(J-t)$ measurements under illumination and short circuit conditions were measured (SI, Figure S7). A fast decay of non-steady state photocurrent observed in device B confirms the efficient charge transport across the device. The characteristics of electron-hole recombination in both devices were obtained from IMVS plot (Figure $3 \mathrm{c}$ and $\mathrm{d}$ ). The values of low and high 


\section{WILEY-VCH}

frequency responses as a function of illumination for device A are in the order of 15 and 0.10 ms, respectively. Whereas for device B, the low frequency response time of $15 \mathrm{~ms}$ with no significant high frequency response is observed.
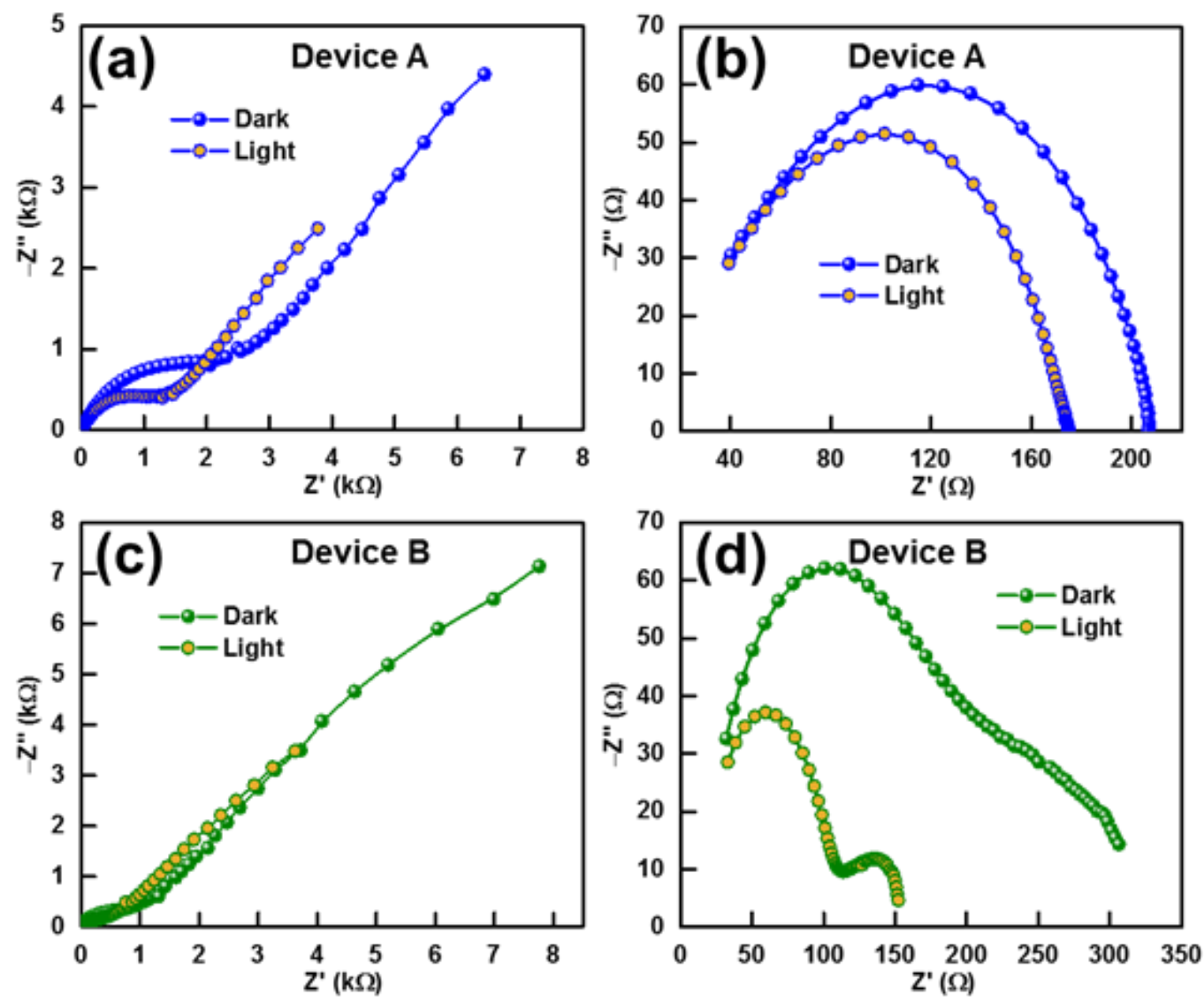

Figure 4. The EIS response measured at (a) $0 \mathrm{~V}$, and (b) at $1.2 \mathrm{~V}$ in dark and under illumination for $\mathrm{CsPbBr}_{3}$ (device A) solar cell, and EIS response measured at (c) $0 \mathrm{~V}$ and (d) $1.3 \mathrm{~V}$ in dark and under illumination for $\mathrm{FAPbBr}_{3}$ (device $\mathrm{B}$ ) solar cell.

Detailed insight into the interfacial processes occurring in the devices based on different absorbers is further gained by EIS (Figure 4 and Figure S2). The EIS spectra were measured in the frequency range of $100 \mathrm{kHz}$ to $1 \mathrm{~Hz}$ with a perturbation voltage of $10 \mathrm{mV}$ in the forward bias range of 0 to $1.4 \mathrm{~V}$ with a step of $50 \mathrm{mV}$. EIS spectra for device $\mathrm{A}$ and $\mathrm{B}$, recorded in dark and under illumination exhibit two clear features: semicircle in the high frequency region followed by an incomplete semicircle arc in the low frequency region. EIS spectra with two or more distinguishable features have also been observed for iodide based 


\section{WILEY-VCH}

PSCs. ${ }^{[11]}$ The EIS spectra corresponding to $\mathrm{CsPbr}_{3}$ recorded at zero bias (Figure 4a) exhibit a maximum value of resistance in the low and high frequency region. With a progressive increase in the applied bias towards the knee voltage $\left(0.9 \mathrm{~V}\right.$ for $\left.\mathrm{CsPbBr}_{3}\right)$, the value of resistance decreased but the values of capacitance increased concurrently. A similar behavior of resistance and capacitance is also observed for the device B shown in Figure $4 \mathrm{c}$ and $\mathrm{d}$. The capacitance of the PSCs at low frequency has been assigned to the ion migration or dielectric relaxation phenomenon. The properties, like giant dielectric constant or change in the magnitude of capacitance with illumination and applied bias are characteristics of perovskite semiconductor mostly reported for iodide based solar cells. ${ }^{[12]}$ We also observed a similar low frequency capacitance in the bromide based PSCs. This infers that the $\mathrm{FAPbBr}_{3}$ and $\mathrm{CsPbBr}_{3}$ systems possess characteristics, which are inherently associated with the absorber layer. The decrease in the low frequency resistance with an increase in the applied bias or illumination for both devices can be explained in the following manner: In the absence of light and bias, $\mathrm{TiO}_{2}$ and perovskite interface exhibits a large electrostatic field of the order of $10^{6} \mathrm{~V} / \mathrm{m}$. The illumination of device causes a significant increase in the majority charge carriers in the absorber layer which leads to the existence of high number of free charge carriers and the interfacial electric field opposing the large electrostatic field. This could explain a decrease in the values of recombination resistance with an increase in the applied bias. Apart from the discussion on the role of illumination and bias on the device characteristics, the above mentioned conclusions also support the assignment of the low frequency arc to the recombination or charge accumulation process. By comparing the low frequency spectra of both devices, a lower magnitude of real complex impedance spectrum in case of device A shows a higher recombination rate. Consequently, with an increase in the applied bias from knee voltage to $V_{O C}$ of a solar cell (Figure $4 \mathrm{~b}$ and Figure SI), magnitude of the low frequency spectrum decreases considerably. Similarly, for device B, the real magnitude of the low frequency spectra decreases by $\sim 100$ times (Figure 4c) in the corresponding bias range. 


\section{WILEY-VCH}

Moreover, at a bias close to the $V_{O C}$ (device A), the low frequency spectrum merges with the high frequency one, whereas at the same time two distinct features of the low and high frequency are still observed for device B (Figure 4d). This signifies the consequence of higher recombination due to photogenerated and bias dependent accumulation of charge carriers in the device A. Between zero to knee voltage for both devices, the high frequency spectra didn't show a significant change in their magnitude. However, a higher absolute value of magnitude at low and high frequency in case of device B further confirms that along with higher recombination, the performance of device A is also limited by the low shunt path.
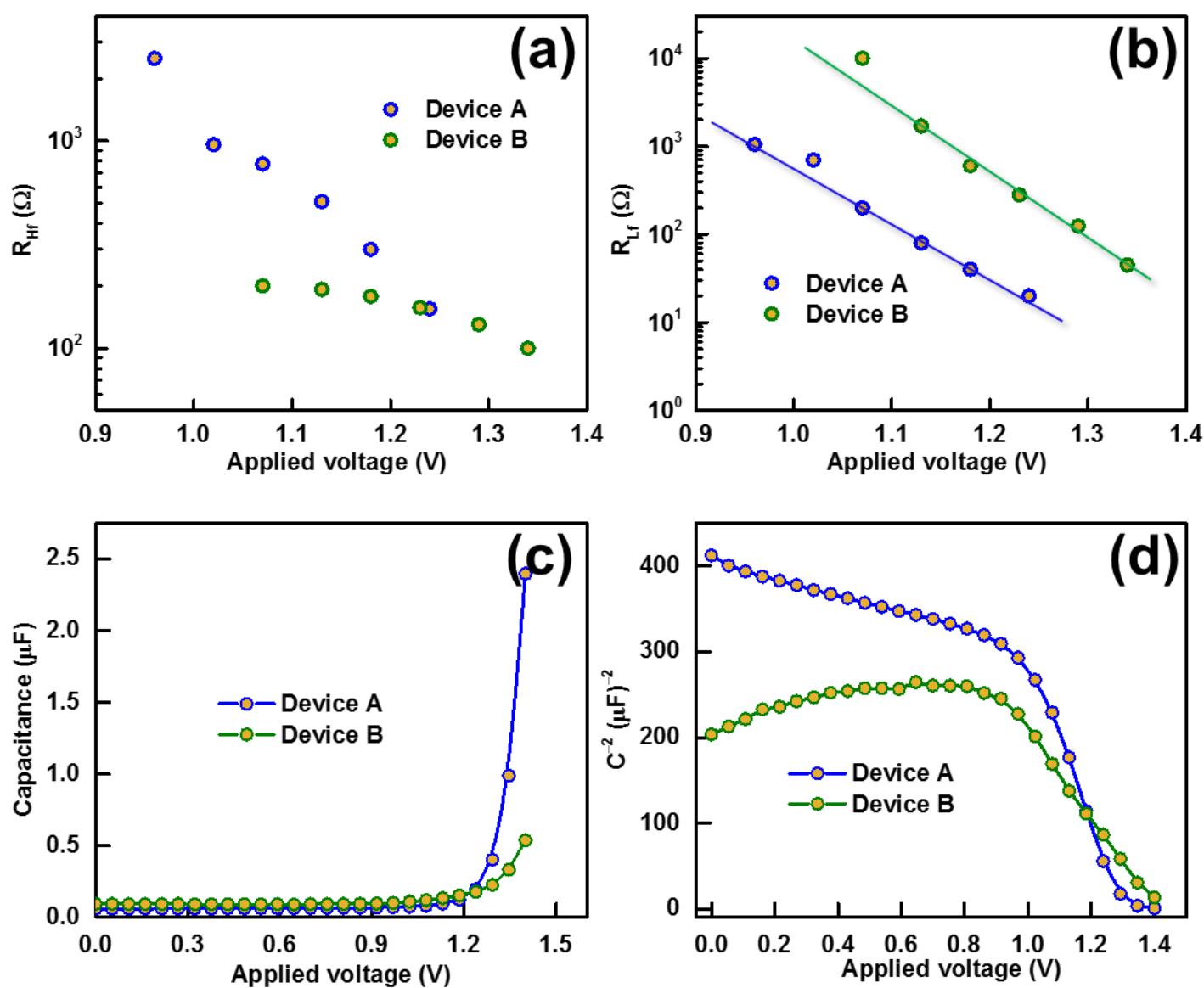

Figure 5. Extracted values from the (a) high and (b) low frequency resistive components of $\mathrm{CsPbBr}_{3}$ (device $\mathrm{A}$ ) and $\mathrm{FAPbBr}_{3}$ (device B) solar cells as a function of applied bias in the range of knee voltage to $V O C$ of corresponding devices, (c) Capacitance versus frequency plot of $\mathrm{CsPbBr}_{3}$ (device A) and $\mathrm{FAPbBr}_{3}$ (device B) solar cells measured at a $10 \mathrm{kHz}$ oscillation frequency and (D) Mott-Schottky plot of $\mathrm{CsPbr}_{3}$ (device A) and $\mathrm{FAPbBr}_{3}$ (device B). 


\section{WILEY-VCH}

Figure $5 \mathrm{a}$ and $\mathrm{b}$ illustrates the extracted values of the high and low frequency resistive components for both devices in a bias range between knee voltage to open circuit voltage. The EIS spectra were fitted by an equivalent circuit of $R_{S}-R_{H f} C_{H f}-R_{L f} C_{L f}$ in which $R_{S}$ is the series resistance, $R_{H f}, R_{L f}$ are the high and low frequency resistive elements, respectively. The $R_{H f}$ and $R_{L f}$ for both devices follow the same bias dependence with somewhat difference in their absolute values. The high frequency $R_{H f}$ is due to the selective contact and is related to the charge transport resistance of holes at perovskite/HTM interface and transport resistance in HTM. $^{[11]}$ A lower values of $R_{H f}$ for device B explains the higher $F F$ because $R_{H f}$ also acts as a series resistance for charge transport. ${ }^{[11 \mathrm{a}]}$ Nevertheless, another parameter that explains the low $F F$ and $V_{O C}$ in device $\mathrm{A}$ is the lower order of $R_{L f}$ as shown in Figure $5 \mathrm{~b}$, where the symbols represents $R_{L f}$ extracted by fitting the EIS spectra and line shows the linear fit according to the expression $\mathrm{R}_{\mathrm{Lf}}=\mathrm{R}_{0} \exp \left[-\mathrm{qV}_{0} / \mathrm{nkT}\right]$. Here, $R_{0}$ is the pre-exponential factor, [11] where straight line fit results in the value of $n$ as 2.0 and 1.88 for device A and B, respectively.

Figure 5c shows the plot of capacitance as a function of applied bias for both devices under illumination. In the low forward bias i.e. between 0 to knee voltage, the values of capacitance remain almost constant with a magnitude of $\sim 0.2 \mu \mathrm{F}$ for both devices. However, in the high forward bias region the capacitance plots exhibit an exponential behavior. Various descriptions have been proposed in literature for the exponential behavior, for e.g. Fermi level modulation of the minority charge carriers in the case of chemical capacitance and charge accumulation capacity of $\mathrm{TiO}_{2} /$ perovskite interface. The fit to the exponential capacitance plot provides an ideality factor which is about $12-15 \%$ lower as compared to the values obtained from the plots shown in Figure 5c. Lower ideality factor from the capacitance-bias plot signifies the different rate of change in capacitance and resistance, or the charge blocking/accumulation nature of the interfacial region. The capacitance as a function of 


\section{WILEY-VCH}

applied bias and frequency are represented by a Mott-Schottky plot for both devices (Figure $5 \mathrm{~d}$ and SI). The slope and intercept provide the values of donor concentration $\left(N_{a}\right)$ and built-in potential $V_{b i}$, respectively. The average value of $V_{b i}$ and $N_{a}$ are obtained as $1.27 \mathrm{~V} \& 1.4 \mathrm{~V}$, and $1.4 \times 10^{16} \& 2.3 \times 10^{16} \mathrm{~cm}^{3}$ for device $\mathrm{A}$ and $\mathrm{B}$, respectively. ${ }^{[13]}$

\section{Conclusions}

Using a combination of techniques, we have successfully unraveled the fundamental electronic processes occurring in the lead bromide based PSCs, which are strongly influenced by the nature of cations present within the inorganic framework. Investigation of the photovoltaic characteristics revealed that the charge transport and interfacial properties are more favorable in $\mathrm{FAPbBr}_{3}$ devices as compared to $\mathrm{CsPbBr} 3$ ones. The small perturbation measurements established that the more rapid charge transport and higher resistive recombination resistance in a relatively more efficient device $(\mathrm{FAPbBr} 3)$ could be ascribed to a decrease in non-radiative recombination. Furthermore, in-depth analysis together with the physical phenomena occurring in the devices allowed us to underline the working principle which will pave a way to further improve the performance of PSCs.

\section{Experimental Section}

Preparation of $\mathrm{TiO}_{2}$ Photoanode. Fluorine-doped tin oxide (FTO) glass substrates (TCO glass, NSG 10, Nippon sheet glass, Japan) were cleaned by ultrasonication in diluted Hellmanex (2\%, in deionized water). The substrates were then rinsed thoroughly with deionized water and ethanol followed by UV/ozone treatment for 15 minutes. A $30 \mathrm{~nm}$ compact layer of titanium dioxide $\left(\mathrm{TiO}_{2}\right)$ was deposited onto the substrate from a precursor solution of titanium diisopropoxide bis(acetylacetonate) solution (75\% in 2- propanol, SigmaAldrich) diluted in anhydrous ethanol (1:9 volume ratio) by spray pyrolysis at $450{ }^{\circ} \mathrm{C}$ using oxygen as the carrying gas. A mesoporous $\mathrm{TiO}_{2}$ layer was then deposited onto the substrate 


\section{WILEY-VCH}

by spin-coating a diluted paste (1:3.5 weight ratio, Dyesol 30NRD) at a speed of $5000 \mathrm{rpm}$ for $30 \mathrm{~s}$ using an acceleration of $2000 \mathrm{rpm}$. This was followed by sintering the substrates at $450{ }^{\circ} \mathrm{C}$ for $30 \mathrm{~min}$ in dry air. For Li-treatment of mesoporous $\mathrm{TiO}_{2}$ photoanode, $200 \mu \mathrm{L}$ of freshly prepared bis(trifluoromethylsulfonyl)imide lithium salt (LiTFSI) solution in acetonitrile $(20 \mathrm{mg} / \mathrm{mL})$ was spin-coated $(3000 \mathrm{rpm}$, acceleration of $2000 \mathrm{rpm}$ for $20 \mathrm{~s})$ after loading time of $10 \mathrm{~s}$. The Li-doped substrates were sintered at $450{ }^{\circ} \mathrm{C}$ for $30 \mathrm{~min}$ and after cooling down to $150{ }^{\circ} \mathrm{C}$ were transferred to a drybox with humidity less than $1 \%$.

Device Fabrication. The $\mathrm{FAPbBr}_{3}$ and $\mathrm{CsPbBr}_{3}$ films were deposited using a two-step sequential deposition method. 1.0 $\mathrm{M} \mathrm{PbBr} 2$ (TCI 99\%) precursor solution was prepared in N,N-dimethylformamide (DMF, Acros Organics) and dimethyl sulfoxide (DMSO, Acros Organics) by constant stirring at $60{ }^{\circ} \mathrm{C}$ for $30 \mathrm{~min} . \mathrm{PbBr}_{2}(\mathrm{DMF}+\mathrm{DMSO}$ in a $1: 1$ volume ratio) solution was spin-coated onto the mesoporous $\mathrm{TiO}_{2}$ films at $3000 \mathrm{rpm}$ for $30 \mathrm{~s}$. This was followed by annealing the films at $80{ }^{\circ} \mathrm{C}$ for $15 \mathrm{~min}$. Then the films were dipped in formamidinium bromide solution (Dyesol, $50 \mathrm{mM}$ ) in isopropanol for $5 \mathrm{~min}$ at $60{ }^{\circ} \mathrm{C}$, rinsed with 2-propanol for $5 \mathrm{~s}$, and dried at $80{ }^{\circ} \mathrm{C}$ for $30 \mathrm{~min}$. For $\mathrm{CsPbBr} 3$ films, $1 \mathrm{M} \mathrm{PbBr} 2$ solution in DMF, kept at $60{ }^{\circ} \mathrm{C}$ during deposition was spin-coated on substrates at $2500 \mathrm{rpm}$ for $30 \mathrm{~s}$ and annealed at $70{ }^{\circ} \mathrm{C}$ for $30 \mathrm{~min}$. A solution of cesium bromide $(\mathrm{CsBr})$ was prepared by dissolving $15 \mathrm{mg} \mathrm{CsBr}$ in $1 \mathrm{~mL}$ methanol at $80{ }^{\circ} \mathrm{C}$ for $10 \mathrm{~min}$ under constant stirring. The $\mathrm{PbBr}_{2}$ substrates were dipped into $\mathrm{CsBr}$ solution at $60{ }^{\circ} \mathrm{C}$ for $10 \mathrm{~min}$, rinsed in isopropanol, and dried at $250{ }^{\circ} \mathrm{C}$ for $10 \mathrm{~min}$.

Deposition of Hole Transport Materials (HTM): $72.3 \mathrm{mg}$ spiro- OMeTAD (2,2',7,7'tetrakis(N,N-di-p-methoxyphenylamine)-9,9-spirobifluorene) was dissolved in $1 \mathrm{~mL}$ of chlorobenzene, to which , $28.8 \mu \mathrm{L}$ of 4-tert-butylpyridine $28.8 \mu \mathrm{L} 17.5 \mu \mathrm{L}$ of a stock solution of $520 \mathrm{mg} \mathrm{mL}^{-1}$ bis(trifluoromethylsulfonyl)imide lithium salt in acetonitrile, and $29 \mu \mathrm{L}$ of a stock solution of $300 \mathrm{mg} \mathrm{mL}^{-1}$ Tris(2-(1H-pyrazol-1-yl)-4-tert-butylpyridine)cobalt-(III) bis(trifluoromethylsulfonyl)imide in acetonitrile were added. The device fabrication was 


\section{WILEY-VCH}

carried out in dry air glove box with humidity $<1 \%$. Finally, device fabrication was completed by thermally evaporating 70nm of gold layer as a back contact.

Device Characterization: The current-voltage (J-V) characteristics of the perovskite devices were recorded with a digital source meter (Keithley model 2400, USA). A 450 W xenon lamp (Oriel, USA) was used as the light source for photovoltaic (J-V) measurements. The spectral output of the lamp was filtered using a Schott K113 Tempax sunlight filter (Präzisions Glas \& Optik GmbH, Germany) to reduce the mismatch between the simulated and actual solar spectrum to less than $2 \%$. The photo-active area of $0.16 \mathrm{~cm}^{2}$ was defined using a dark-coloured metal mask. AC measurements were performed using a potentiostat Biologic SP300 equipped with a frequency response analyser. IS measurements were performed in the DC bias range of 0 to open circuit voltage with an $\mathrm{AC}$ perturbation signal of $20 \mathrm{mV}$ in the frequency range of $1 \mathrm{~Hz}$ to $100 \mathrm{kHz}$.

\section{Supporting Information}

Supporting Information is available from the Wiley Online Library or from the author.

\section{Acknowledgements}

P.Y. gratefully acknowledges financial support from the Swiss Confederation under Swiss Government Scholarship. M.I.D, S.M.Z and M.G. thank the King Abdulaziz City for Science and Technology (KACST), the European Union's Horizon 2020 programme through a FET Open research and innovation action under grant agreement No 687008 and Swiss National Science Foundation (SNSF) for financial support.

Received: ((will be filled in by the editorial staff))

Revised: ((will be filled in by the editorial staff)) Published online: ((will be filled in by the editorial staff))

\section{References}

[1] a) A. Kojima, K. Teshima, Y. Shirai, T. Miyasaka, J. Am. Chem. Soc. 2009, 131, 6050. b) H-S. Kim, C-R. Lee, J-H. Im, K-B. Lee, T. Moehl, A. Marchioro, S-J. Moon, R. Humphry-Baker, J-H. Yum, J. E. Moser, M. Grätzel, N-G. Park, Sci. Rep. 2012, 2, 


\section{WILEY-VCH}

591:1. c) M. M. Lee, J. Teuscher, T. Miyasaka, T. N. Murakami, H. J. Snaith, Science 2012, 338, 643. d) C. Quarti, E. Mosconi, J. M. Ball, V. D'Innocenzo, C. Tao, S. Pathak, H. J. Snaith, A. Petrozza, F. De Angelis, Energ. Env. Sci. 2016, 9, 155; e) E. Edri, S. Kirmayer, A. Henning, S. Mukhopadhyay, K. Gartsman, Y. Rosenwaks, G. Hodes, D. Cahen, Nano Lett. 2014, 14, 1000; f) S. D. Stranks, G. E. Eperon, G. Grancini, C. Menelaou, M. J. P. Alcocer, T. Leijtens, L. M. Herz, A. Petrozza, H. J. Snaith, Science 2013, 342, 341; g) G. Xing, N. Mathews, S. Sun, S. S. Lim, Y. M. Lam, M. Grätzel, S. Mhaisalkar, T. C. Sum, Science 2013, 342, 344.

[2] a) W. S. Yang, J. H. Noh, N. J. Jeon, Y. C. Kim, S. Ryu, J. Seo, S. I. Seok, Science 2015, 348, 1234; b) W. S. Yang, B.-W. Park, E. H. Jung, N. J. Jeon, Y. C. Kim, D. U. Lee, S. S. Shin, J. Seo, E. K. Kim, J. H. Noh, S. I. Seok, Science 2017, 356, 1376.

[3] a) K. Galkowski, A. Mitioglu, A. Miyata, P. Plochocka, O. Portugall, G. E. Eperon, J. T.-W. Wang, T. Stergiopoulos, S. D. Stranks, H. J. Snaith, R. J. Nicholas, Energ. Environ. Sci. 2016, 9, 962; b) G. E. Eperon, V. M. Burlakov, P. Docampo, A. Goriely, H. J. Snaith, Adv. Funct. Mater. 2014, 24, 151; c) Z.-K. Tan, R. S. Moghaddam, M. L. Lai, P. Docampo, R. Higler, F. Deschler, M. Price, A. Sadhanala, L. M. Pazos, D. Credgington, F. Hanusch, T. Bein, H. J. Snaith, R. H. Friend, Nat. Nanotechnol. 2014, 9, 687.

[4] a) F. C. Hanusch, E. Wiesenmayer, E. Mankel, A. Binek, P. Angloher, C. Fraunhofer, N. Giesbrecht, J. M. Feckl, W. Jaegermann, D. Johrendt, T. Bein, P. Docampo, J. Phys. Chem. Lett. 2014, 5, 2791; b) N. Arora, S. Orlandi, M. I. Dar, S. Aghazada, G. Jacopin, M. Cavazzini, E. Mosconi, P. Gratia, F. De Angelis, G. Pozzi, M. Graetzel, M. K. Nazeeruddin, ACS Energ. Lett. 2016, 1, 107.

[5] a) N. Arora, M. I. Dar, M. Abdi-Jalebi, F. Giordano, N. Pellet, G. Jacopin, R. H. Friend, S. M. Zakeeruddin, M. Grätzel, Nano Lett. 2016, 16, 7155; b) M. Kulbak, D. Cahen, G. Hodes, J. Phys. Chem. Lett. 2015, 6, 2452; c) Q. A. Akkerman, M. Gandini, 


\section{WILEY-VCH}

F. Di Stasio, P. Rastogi, F. Palazon, G. Bertoni, J. M. Ball, M. Prato, A. Petrozza, L. Manna, Nat. Energ. 2016, 2, 16194.

[6] P. Yadav, K. Pandey, P. Bhatt, D. Raval, B. Tripathi, C. Kanth P, M. K. Pandey, M. Kumar, Sol. Energ. 2015, 122, 773.

[7] T. Hahn, S. Tscheuschner, F.-J. Kahle, M. Reichenberger, S. Athanasopoulos, C. Saller, G. C. Bazan, T.-Q. Nguyen, P. Strohriegl, H. Bässler, A. Köhler, Adv. Funct. Mater. 2016, DOI: 10.1002/adfm.201604906n/a.

[8] D. Bi, W. Tress, M. I. Dar, P. Gao, J. Luo, C. Renevier, K. Schenk, A. Abate, F. Giordano, J.-P. Correa Baena, J.-D. Decoppet, S. M. Zakeeruddin, M. K. Nazeeruddin, M. Grätzel, A. Hagfeldt, Sci. Adv. 2016, 2.

[9] a) H. D. Kim, H. Ohkita, H. Benten, S. Ito, Adv. Mater. 2016, 28, 917; b) Y. Galagan, E. W. C. Coenen, W. J. H. Verhees, R. Andriessen, J. Mater. Chem. A 2016, 4, 5700.

[10] a) F. Giordano, A. Abate, J. P. Correa Baena, M. Saliba, T. Matsui, S. H. Im, S. M. Zakeeruddin, M. K. Nazeeruddin, A. Hagfeldt, M. Graetzel, Nat. Comm. 2016, 7, 10379; b) E. Guillén, F. J. Ramos, J. A. Anta, S. Ahmad, J. Phys. Chem. C 2014, 118, 22913.

[11] a) A. Guerrero, G. Garcia-Belmonte, I. Mora-Sero, J. Bisquert, Y. S. Kang, T. J. Jacobsson, J.-P. Correa-Baena, A. Hagfeldt, J. Phys. Chem. C 2016, 120, 8023; b) J. Bisquert, G. Garcia-Belmonte, I. Mora-Sero, in Unconventional Thin Film Photovoltaics, DOI: 10.1039/9781782624066-00057, The Royal Society of Chemistry 2016, p. 57.

[12] a) S. Shao, M. Abdu-Aguye, L. Qiu, L.-H. Lai, J. Liu, S. Adjokatse, F. Jahani, M. E. Kamminga, G. H. ten Brink, T. T. M. Palstra, B. J. Kooi, J. C. Hummelen, M. Antonietta Loi, Energ. Environ. Sci. 2016, 9, 2444; b) G. Garcia-Belmonte, J. Bisquert, ACS Energ. Lett. 2016, 1, 683; c) M. Valles-Pelarda, B. C. Hames, I. García- 


\section{WILEY-VCH}

Benito, O. Almora, A. Molina-Ontoria, R. S. Sánchez, G. Garcia-Belmonte, N. Martín, I. Mora-Sero, J. Phys. Chem. Lett. 2016, 7, 4622.

[13] a) T. Leijtens, G. E. Eperon, A. J. Barker, G. Grancini, W. Zhang, J. M. Ball, A. R. S. Kandada, H. J. Snaith, A. Petrozza, Energ. Environ. Sci. 2016, 9, 3472; b) H. Yu, H. Lu, F. Xie, S. Zhou, N. Zhao, Adv. Funct. Mater. 2016, 26, 1411.

We explored the effect of cations on the intrinsic and interfacial dynamic processes occurring in the perovskite solar cells which allowed us to underline their working principle

Keyword: (perovskite solar cell, organic cation, interfacial dynamics, electrochemical impedance spectroscopy, current voltage characteristics)

Pankaj Yadav, Mohammad Alotaibi, Neha Arora, M. Ibrahim Dar,* Shaik Mohammed Zakeeruddin, and Michael Grätzel

Influence of the nature of A cation on dynamics of charge transfer processes in perovskite solar cells

ToC figure

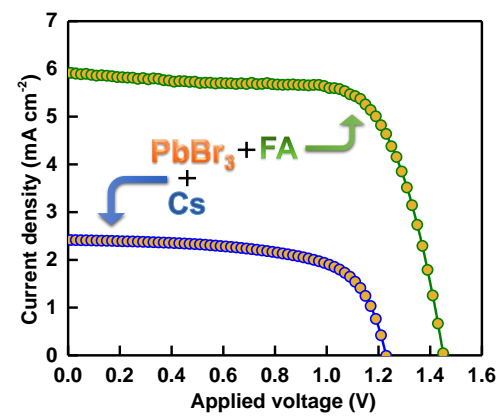

